\title{
Pharmacokinetic Evaluation of the Interactions of Amenamevir (ASP2151) with Ketoconazole, Rifampicin, Midazolam, and Warfarin in Healthy Adults
}

Tomohiro Kusawake · Martin den Adel • Dorien Groenendaal-van de Meent •

Alberto Garcia-Hernandez • Akitsugu Takada · Kota Kato • Yoshiaki Ohtsu •

Masataka Katashima

Received: September 1, 2017 / Published online: October 26, 2017

(C) The Author(s) 2017. This article is an open access publication

\section{ABSTRACT}

Introduction: Amenamevir is a nonnucleoside antiherpes virus compound available for treating herpes zoster infections. Four studies aimed to determine any potential interactions between amenamevir and ketoconazole, rifampicin, midazolam, or warfarin in healthy male participants.

Methods: Two studies were open-label studies that evaluated the effects of multiple doses of ketoconazole (400 mg) and rifampicin (600 mg) on the pharmacokinetics of a single oral dose of amenamevir. The other two studies were randomized, double-blind, parallel-group studies that evaluated the effects of multiple doses of amenamevir on the pharmacokinetics of a

Enhanced content To view enhanced content for this article go to http://www.medengine.com/Redeem/ C8CCF060639C490D.

T. Kusawake $(\bowtie)$

Astellas Pharma Global Development Inc.,

Northbrook, IL, USA

e-mail: tomohiro.kusawake@astellas.com

M. den Adel · D. Groenendaal-van de Meent .

A. Garcia-Hernandez

Astellas Pharma Europe B.V., Leiden, The

Netherlands

A. Takada $\cdot$ M. Katashima

Astellas Pharma Inc., Tokyo, Japan

K. Kato - Y. Ohtsu

Astellas Pharma Inc., Tsukuba, Japan single dose of midazolam $(7.5 \mathrm{mg})$ and warfarin (25 mg). A drug interaction was considered to occur if the $90 \%$ confidence interval (CI) of the least squares geometric mean ratio (GMR) of amenamevir to the comparator was outside the prespecified interval of 0.80-1.25.

Results: Interactions were observed between amenamevir and ketoconazole, rifampicin, and midazolam, but not between amenamevir and warfarin. After a single 400-mg dose of amenamevir, the GMRs of amenamevir plus ketoconazole or rifampicin versus amenamevir alone for $C_{\max }$ and the area under the plasma concentration-time curve from time zero to infinity $\left(\mathrm{AUC}_{\mathrm{inf}}\right)$ were 1.30 (90\% CI 1.17-1.45) and 2.58 (90\% CI 2.32-2.87), respectively, for ketoconazole and 0.42 (90\% CI 0.37-0.49) and 0.17 (90\% CI 0.15-0.19), respectively, for rifampicin. Following multiple doses of amenamevir (400 mg), the GMRs of midazolam plus amenamevir versus midazolam alone for $\mathrm{AUC}_{\mathrm{inf}}$ and $C_{\max }$ were 0.53 (90\% CI 0.47-0.61) and 0.63 (90\% CI 0.50-0.80), respectively. After a single dose of warfarin, the $(S)$-warfarin and $(R)$-warfarin mean $C_{\max }$ increased and mean $\mathrm{AUC}_{\mathrm{inf}}$ decreased in the presence of amenamevir; however, the $90 \%$ CIs of the GMRs for these parameters remained within the predefined limits.

Conclusion: These findings confirm that amenamevir (as a cytochrome P450 3A4 substrate) can interact with ketoconazole or rifampicin, and (as a cytochrome P450 3A4 inducer) can 
interact with midazolam; however, no interaction between amenamevir and (S)-warfarin was observed, indicating that amenamevir is not an inducer of cytochrome P450 2C9.

Funding: Astellas Pharma.

Trial registration: EudraCT2007-002227-33 (study 15L-CL-008), EudraCT2007-002228-14 (study 15L-CL-009), EudraCT2007-002761-13 (study 15L-CL-010), and EudraCT2007-00277914 (study 15L-CL-018).

Keywords: Amenamevir; Drug-drug interactions; Ketoconazole; Midazolam; Pharmacokinetics; Rifampicin; Safety; Varicella-zoster virus; Warfarin

\section{INTRODUCTION}

Varicella-zoster virus (VZV) is a human herpesvirus (HHV) that leads to the development of two distinct diseases: varicella (chicken pox) as the first episode and herpes zoster (shingles) as the recurrent episode [1]. VZV is neurotrophic and remains latent in dorsal sensory ganglia after the initial infection [2]. Herpes zoster is a manifestation of the reactivation of latent VZV. It is relatively uncommon in immunocompetent individuals younger than 40 years, but the incidence of herpes zoster markedly increases in those older than 50 years [3]. While the incidence rate of developing herpes zoster is $6-8$ per 1000 person-years at 60 years of age, the incidence rate in patients aged 80 years or older increases to 8-12 per 1000 person-years [3].

Nucleoside analogs such as acyclovir [4], valacyclovir [5], and famciclovir [6] have been approved for the treatment of herpes simplex virus 1 , herpes simplex virus 2 , and VZV infections. Although these drugs are used for the treatment of HHV infections, there is a medical need for therapies with improved antiviral activity to provide more rapid and complete resolution of signs and symptoms.

Amenamevir (ASP2151) is a nonnucleoside antiherpes compound developed for the treatment of VZV infections. Preclinical studies have indicated that amenamevir targets the viral helicase-primase complex, which is essential for viral DNA replication $[7,8]$. The main elimination pathway of amenamevir was hepatic metabolism in animal studies (unpublished data). In a study in healthy volunteers, urinary excretion as unchanged drug was approximately $10 \%$ [9]. In vitro data further clarified the main elimination pathway; the metabolism of amenamevir is significantly correlated with marker-enzyme activities specific for the cytochrome P450 (CYP) isozymes CYP2B6, CYP2C19, and CYP3A4/5 [10]. The strongest correlation was observed for CYP3A4/5 $\left(r^{2}=0.9236, p<0.0001\right)$, suggesting CYP3A4/ 5 -mediated metabolism is the main pathway for elimination of amenamevir. In addition, in vitro enzyme induction data indicated that amenamevir potentially induces CYP3A4 and CYP2C9 activities, with a weak potential for inducing CYP2C19 (unpublished data).

Herpes zoster is a major disease of the elderly population, and patients often require concomitant medications for the treatment of other comorbidities [11]. Therefore, it is important to determine any potential interactions between medications whose metabolism is mediated by CYP3A4 and CYP2C9.

This article summarizes the results of four drug-drug interaction phase 1 studies that investigated potential interactions between amenamevir and ketoconazole (a strong CYP3A4 inhibitor), rifampicin (a strong CYP3A4 inducer), midazolam (a CYP3A4 substrate), and (S)-warfarin (a CYP2C9 substrate) in healthy adults.

\section{METHODS}

\section{Study Design}

Each of the four drug-drug interaction studies was conducted at a single study site in France by the contract research organization SGS-Aster. Study 15L-CL-008 (EudraCT2007-002227-33) was conducted between October 17, 2007, and November 28, 2007, study 15L-CL-009 (EudraCT2007-002228-14) was conducted between October 19, 2007, and November 28, 2007, study 15L-CL-010 (EudraCT2007-002761-13) was conducted between September 13, 2007, and November 17, 2007, and study 15L-CL-018 (EudraCT2007-002779-14) was conducted 
between September 13, 2007, and December 3, 2007. Two of the studies were open-label studies that evaluated the effects of multiple doses of ketoconazole (study 15L-CL-008) and rifampicin (study 15L-CL-009) on the pharmacokinetics of a single oral dose of amenamevir. The other two studies were randomized, double-blind, parallel-group studies that evaluated the effects of multiple doses of amenamevir (200 and $400 \mathrm{mg}$ ) on the pharmacokinetics of a single dose of midazolam (study 15L-CL-010) and a single dose of warfarin (study 15L-CL-018).

The study protocols were approved by the institutional review boards at the study site [Hôpital Ambroise-Paré, Secrétariat du CPP Ile de France VIII, Laboratoire d'Anatomopathologie (studies 15L-CL-008 and 15L-CL-009) and CPP Ile de France I, Secrétariat du CPP Ile de France I, Hôpital Hotel-Dieu (studies 15L-CL-010 and 15L-CL-018)]. All procedures followed were in accordance with the ethical standards of the responsible committee on human experimentation (institutional and national) and with the Helsinki Declaration of 1964, as revised in 2000. Informed consent was obtained from all participants for their being included in the study.

\section{Study Participants}

All four studies enrolled healthy male adults (aged 18-55 years). Studies 15L-CL-008 and 15L-CL-009 required participants to have a body weight of at least $60 \mathrm{~kg}$ but less than $100 \mathrm{~kg}$ and a body mass index of at least $18 \mathrm{~kg} / \mathrm{m}^{2}$ but less than $30 \mathrm{~kg} / \mathrm{m}^{2}$. Studies 15L-CL-010 and 15L-CL-018 required participants to have a body mass index of at least $18 \mathrm{~kg} / \mathrm{m}^{2}$ but less than $30 \mathrm{~kg} / \mathrm{m}^{2}$. The exclusion criteria included, but were not limited to, the following: a known or suspected hypersensitivity to any of the study drugs or any of the components of the formulations used; any clinically significant history of asthma, eczema, or any other allergic or severe hypersensitivity to any drug; a history of clinically significant electrocardiogram (ECG) abnormalities or any clinically relevant history of other diseases or disorders, including cardiovascular, gastrointestinal, respiratory, renal, hepatic, neurological, dermatological, psychiatric, or metabolic disorders, as judged by the study investigator. Study 15L-CL-018 excluded participants with a history of and/or signs and symptoms of current abnormal hemostasis or blood dyscrasia or abnormal prothrombin time (PT) or activated partial thromboplastin time at screening.

\section{Dosing and Sampling Schedules}

In study 15L-CL-008, participants received a single oral dose of amenamevir (400 $\mathrm{mg}$ as two 200-mg tablets) on day 1, after which they underwent a 48 -h washout period. They then received ketoconazole orally $(400 \mathrm{mg}$ as two $200-\mathrm{mg}$ tablets) once daily on days $3-13$, with a second single dose of amenamevir ( $400 \mathrm{mg}$ ) administered in combination with ketoconazole on day 10. Blood samples for amenamevir analysis were collected before dosing and 30 min and 1, 1.5, 2, 3, 4, 5, 6, 8, 12, 16, 24, and $36 \mathrm{~h}$ after dosing on day 1 , before dosing on day 3 , and before dosing and $30 \mathrm{~min}$ and $1,1.5,2,3$, $4,5,6,8,12,16,24,36,48,60,72,84$, and $96 \mathrm{~h}$ after dosing on day 10.

In study 15L-CL-009, participants received a single oral dose of amenamevir (400 mg) on day 1 and underwent a 48-h washout period, after which they received rifampicin orally (600 $\mathrm{mg}$ as two 300-mg tablets) once daily on days $3-11$, with a second single dose of amenamevir (400 mg) administered with rifampicin on day 10. Blood samples for amenamevir analysis were collected before dosing and $30 \mathrm{~min}$ and $1,1.5,2,3,4,5,6,8,12,16,24$, and $36 \mathrm{~h}$ after dosing on day 1 , before dosing on day 3 , and before dosing and $30 \mathrm{~min}$ and 1 , $1.5,2,3,4,5,6,8,12,16,24,36$, and $48 \mathrm{~h}$ after dosing on day 10 .

In study 15L-CL-010, participants were randomly assigned to receive amenamevir orally ( 200 or $400 \mathrm{mg}$ ) once daily on days $3-12$, and all participants received a single dose of midazolam (7.5 mg as one 7.5-mg tablet) on days 1 and 12 . Blood samples for analysis of midazolam were collected before dosing and 15 and $30 \mathrm{~min}$ and $1,1.5,2,3,4,5,6,8,12,16,24$, and $36 \mathrm{~h}$ after dosing on days 1 and 12 . 
Study 15L-CL-018 had two treatment periods: in period 1 , participants received a single oral dose of warfarin ( $25 \mathrm{mg}$; five $5-\mathrm{mg}$ tablets) on day 1 , followed by a 2 -week washout period; in period 2, participants were randomly assigned to receive amenamevir (200 or $400 \mathrm{mg}$ ) once daily on days $1-17$, and all participants received warfarin $(25 \mathrm{mg})$ in combination with amenamevir on day 10. Blood samples for warfarin analysis were obtained before dosing and $1,2,4,6,8,12,16,24,36,48,60,72,96,120$, 144 , and $168 \mathrm{~h}$ after the first single dose of warfarin on day 1 of period 1 , and on day 10 of period 2, and PT analysis was performed before dosing and 1, 4, 8, 12, 24, 48, 72, 120, and $168 \mathrm{~h}$ after the first single dose of warfarin on day 1 of period 1 , and on day 10 of period 2 .

In all four studies, amenamevir was administered under fed conditions. In studies 15L-CL-008, 15L-CL-010, and 15L-CL-018, amenamevir and the comparator were both administered with $240 \mathrm{~mL}$ of water within $5 \mathrm{~min}$ after a standardized breakfast. In study 15L-CL-009, rifampicin was administered with $120 \mathrm{~mL}$ of water $1 \mathrm{~h}$ before the participants ate a standardized breakfast, and amenamevir was administered with $120 \mathrm{~mL}$ of water within $5 \mathrm{~min}$ after breakfast. Participants drank $120 \mathrm{~mL}$ of water $1 \mathrm{~h}$ before breakfast on day 1 for consistency with day-10 dosing. Amenamevir was administered with food on the basis of the results of a phase I study in healthy volunteers that showed that the pharmacokinetics of amenamevir were affected by food, with the area under the plasma concentration-time curve (AUC) from time zero to infinity $\left(A U C_{i n f}\right)$ almost doubling when amenamevir was administered with food [12].

In studies 15L-CL-010 and 15L-CL-018, blinding of the amenamevir dose was maintained by administration of one active 200-mg amenamevir tablet and one matching placebo to participants assigned to receive amenamevir at a dose of $200 \mathrm{mg}$, and administration of two active 200-mg amenamevir tablets to those randomized to receive amenamevir at a dose of $400 \mathrm{mg}$.

\section{Pharmacokinetic Assessments}

Plasma concentrations of amenamevir, midazolam, $(S)$-warfarin, and $(R)$-warfarin were determined by validated liquid chromatography-tandem mass spectrometry methods. The amenamevir method included direct plasma injection (MAYI-ODS trapping column), followed by reverse phase (Xterra MS $\mathrm{C}_{18}$, $30 \mathrm{~mm} \times 4.6 \mathrm{~mm}$ ) liquid chromatography with tandem mass spectrometry detection (TSQ Quantum with atmospheric pressure chemical ionization interface in negative mode). The method is validated over a range of $5-10,000 \mathrm{ng} / \mathrm{mL}$ with $0.2 \mathrm{~mL}$ plasma. The midazolam method involved a liquid-liquid extraction with methyl tert-butyl ether at $\mathrm{pH} 10$, followed by reverse phase (Chromolith Performance, RP-18e, $100 \mathrm{~mm} \times 4.6 \mathrm{~mm})$ liquid chromatography with tandem mass spectrometry detection (API3000 with atmospheric pressure chemical ionization interface in positive mode). The method is validated over a range of $0.1-100 \mathrm{ng} / \mathrm{mL}$ with $0.25 \mathrm{~mL}$ plasma. The warfarin method involved a solid-phase extraction on Oasis HLB 1 cc 30-mg cartridges, followed by chiral (CHIRALCEL ODR-L $250 \mathrm{~mm}$ x $4.6 \mathrm{~mm}$ ) liquid chromatography with tandem mass spectrometry detection (API3000 with electrospray ionization interface in negative mode). The method is validated over a range of 5-2500 ng/mL with $0.1 \mathrm{~mL}$ plasma.

The pharmacokinetic parameters assessed included plasma maximum concentration $\left(C_{\max }\right), \mathrm{AUC}_{\mathrm{inf}}$, time to plasma $C_{\max }\left(t_{\max }\right)$, plasma half-life $\left(t_{1 / 2}\right)$, total plasma clearance $(\mathrm{CL} / \mathrm{F})$. In all four studies, the least squares (LS) geometric mean ratios (GMRs) of $C_{\max }$ and $\mathrm{AUC}_{\text {inf }}$ were also calculated, and if the $90 \%$ confidence interval (CI) for both $C_{\max }$ and $\mathrm{AUC}_{\text {inf }}$ was within the prespecified interval of $0.80-1.25$, the absence of significant interaction between amenamevir and ketoconazole (study 15L-CL-008), amenamevir and rifampicin (study 15L-CL-009), amenamevir and midazolam (study 15L-CL-010), or amenamevir and warfarin (study 15L-CL-018) was concluded. 


\section{Pharmacodynamic Assessments}

In study 15L-CL-018, the pharmacodynamic assessments for warfarin included AUC for PT from time zero to $168 \mathrm{~h}$ (AUC $\mathrm{PT}_{\mathrm{PT}} \mathrm{0}-168 \mathrm{~h}$ ), maximum PT $\left(\mathrm{PT}_{\max }\right)$, and time to reach $\mathrm{PT}_{\max }$ $\left(t_{\mathrm{PT} ; \max }\right)$. A significant interaction between amenamevir and warfarin was also concluded if the $90 \%$ CI for the LS GMR of AUC $\mathrm{PT}_{\mathrm{PT}-168 \mathrm{~h} \text { and }}$ $\mathrm{PT}_{\max }$ was outside the prespecified interval of $0.80-1.25$.

\section{Safety Assessments}

In all four studies, safety assessments included the evaluation of the frequency and severity of treatment-emergent adverse events (TEAEs), 12-lead ECG recordings, clinical laboratory parameters (biochemistry, hematology, serology, and urinalysis), vital signs (blood pressure and pulse), and physical examination findings. TEAEs were coded according to the Medical Dictionary for Regulatory Activities version 9.1 (study 15L-CL-010) or version 10.1 (remaining studies), and were summarized by system organ class and preferred term.

\section{Statistical Analysis}

Statistical analysis was conducted by Astellas Pharma. For studies 15L-CL-008 and 15L-CL-009, the sample sizes needed to evaluate the interaction between amenamevir and ketoconazole or rifampicin were calculated with an assumed coefficient of variation for the AUC ratio of $18.45 \%$ and for the $C_{\max }$ ratio of $21.40 \%$, so the $90 \%$ CI for the treatment ratio had a multiplicative distance from the ratio to the outer limits of 1.125 . From these calculations, the number of participants required was 20; given an assumed drop-out rate of $8-10 \%$, a total of 22 participants were planned and recruited for each of these studies. For studies 15L-CL-010 and 15L-CL-018, the sample sizes needed to demonstrate the absence of a clinically relevant interaction between amenamevir and midazolam or warfarin were calculated with an assumed coefficient of variation of $19.4 \%$ (for midazolam $C_{\max }$ ) or $15.6 \%$ (for warfarin
$\left.C_{\max }\right)$ and a power of $85 \%$ or $90 \%$, respectively, for each individual test. Assuming a weak induction effect, so that the $90 \%$ CI for the treatment ratio ranged from $80 \%$ to $125 \%$, the number of participants required for each amenamevir dose group (200 and $400 \mathrm{mg}$ ) was 20 (study 15L-CL-010) or 16 (study 15L-CL-018). Assuming a drop-out rate of $8-10 \%, 22$ participants per dose group were recruited in study 15L-CL-010, and 18 participants per dose group were recruited in study 15L-CL-018.

Calculations were performed with actual sampling times by noncompartmental methods with use of WinNonlin ${ }^{\circledR}$ Professional version 5.0 or higher (Pharsight, Mountain View, CA, USA) or SAS ${ }^{\circledR}$ version 8.2 or higher (SAS Institute, Cary, NC, USA). Studies 15L-CL-008, 15L-CL-009, and 15L-CL-010 had two analysis set populations: the safety analysis set (SAF; defined as all participants who received at least one dose of the study drug) and the pharmacokinetics analysis set (PKAS; defined as all participants in the SAF population for whom sufficient plasma concentration data were available to provide at least one pharmacokinetic parameter). In addition to the SAF and PKAS populations, study 15L-CL-018 included a pharmacodynamic analysis set, which included all participants from the SAF population for whom sufficient plasma concentration data were available to provide at least one pharmacodynamic parameter.

For each study, absence of a clinically significant pharmacokinetic drug-drug interaction was concluded if the $90 \% \mathrm{CI}$ for the both the $\mathrm{AUC}_{\text {inf }}$ ratio and the $C_{\max }$ ratio fell within the prespecified interval of $0.80-1.25$ according to the following hypothesis testing:

- HO (null hypothesis): $\mathrm{AUC}_{\text {inf }}$ ratio less than 0.80 or greater than 1.25 .

- H1 (alternative hypothesis): $\mathrm{AUC}_{\mathrm{inf}}$ ratio 0.80 or greater and 1.25 or less.

Identical hypothesis testing were performed for $C_{\max }$. $\mathrm{AUC}_{\mathrm{inf}}$ and $C_{\max }$ were logarithmically transformed (natural logarithm) and then subjected to a general linear model including period and participant as fixed factors..

In study 15L-CL-018, the same hypothesis testing was performed for the pharmacodynamic parameters $\mathrm{AUC}_{\mathrm{PT} ; 0-168 \mathrm{~h}}$ and $\mathrm{PT}_{\max }$. 
Table 1 Summary of baseline characteristics

\begin{tabular}{|c|c|c|c|c|c|c|}
\hline & \multicolumn{6}{|l|}{ Study } \\
\hline & \multirow{2}{*}{$\begin{array}{l}\text { 15L-CL-008 } \\
(n=22)\end{array}$} & \multirow{2}{*}{$\begin{array}{l}15 \mathrm{~L}-\mathrm{CL}-009 \\
(n=22)\end{array}$} & \multicolumn{2}{|l|}{ 15L-CL-010 } & \multicolumn{2}{|l|}{ 15L-CL-018 } \\
\hline & & & $\begin{array}{l}\text { Amenamevir } \\
200 \mathrm{mg} \\
(n=22)\end{array}$ & $\begin{array}{l}\text { Amenamevir } \\
400 \mathrm{mg} \\
(n=22)\end{array}$ & $\begin{array}{l}\text { Amenamevir } \\
200 \mathrm{mg} \\
(n=18)\end{array}$ & $\begin{array}{l}\text { Amenamevir } \\
400 \mathrm{mg} \\
(n=19)\end{array}$ \\
\hline Age (years) & $30.0 \pm 8.4$ & $31.9 \pm 7.9$ & $36.3 \pm 9.5$ & $34.1 \pm 10.9$ & $30.3 \pm 9.4$ & $29.5 \pm 8.5$ \\
\hline \multicolumn{7}{|l|}{ Race } \\
\hline White & $13(59.1 \%)$ & $16(72.7 \%)$ & $16(72.7 \%)$ & $18(81.8 \%)$ & $13(72.2 \%)$ & $12(63.2 \%)$ \\
\hline $\begin{array}{l}\text { Black or } \\
\text { African } \\
\text { American }\end{array}$ & $6(27.3 \%)$ & $3(13.6 \%)$ & $6(27.3 \%)$ & $3(13.6 \%)$ & $4(22.2 \%)$ & $3(15.8 \%)$ \\
\hline Asian & 0 & 0 & 0 & 0 & 0 & 0 \\
\hline Other & $3(13.6 \%)$ & $3(13.6 \%)$ & 0 & $1(4.5 \%)$ & $1(5.6 \%)$ & $4(21.1 \%)$ \\
\hline Weight $(\mathrm{kg})$ & $78.2 \pm 10.6$ & $77.6 \pm 8.9$ & $74.2 \pm 11.1$ & $74.6 \pm 9.1$ & $74.0 \pm 8.8$ & $77.7 \pm 9.3$ \\
\hline Height (m) & $1.77 \pm 0.05$ & $1.76 \pm 0.06$ & $1.75 \pm 0.05$ & $1.75 \pm 0.05$ & $1.77 \pm 0.05$ & $1.79 \pm 0.05$ \\
\hline $\operatorname{BMI}\left(\mathrm{kg} / \mathrm{m}^{2}\right)$ & $25.0 \pm 2.5$ & $25.1 \pm 2.7$ & $24.1 \pm 3.2$ & $24.3 \pm 2.8$ & $23.6 \pm 2.6$ & $24.3 \pm 2.6$ \\
\hline
\end{tabular}

All values are presented as the mean \pm standard deviation unless stated otherwise

$B M I$ body mass index

\section{RESULTS}

\section{Study Population}

Studies 15L-CL-008 and 15L-CL-009 both enrolled a total of 22 healthy male participants, all of whom received the study drug, completed the study, and were included in the SAF and PKAS populations. In study 15L-CL-010, 44 male participants were randomized and treated, and all participants completed the study and were included in the SAF and PKAS populations. In study 15L-CL-018, 37 male participants were randomized and treated, and 35 participants completed the study. In this study, two participants prematurely discontinued their participation in the study after receiving amenamevir at a dosage of $400 \mathrm{mg}$ once daily for 3 and 8 days, respectively, because of withdrawal of consent; therefore, the SAF population consisted of 37 participants and the PKAS population consisted of 35 participants. There were no
Fig. 1 Mean amenamevir plasma concentration versus time curve after administration of a single 400-mg dose of amenamevir a alone (open circles) and with ketoconazole at a dosage of $400 \mathrm{mg}$ once daily (closed circles), and $\mathbf{b}$ alone (open circles) and with rifampicin at a dosage of $600 \mathrm{mg}$ once daily (closed circles)

deviations in study drug adherence in study 15L-CL-008, 15L-CL-009, or 15L-CL-010.

The demographics and baseline characteristics of the participants in the four studies are summarized in Table 1.

\section{Pharmacokinetics}

\section{Effects of Ketoconazole on Amenamevir Pharmacokinetics}

After a single 400-mg dose of amenamevir in study 15L-CL-008, the amenamevir mean $C_{\max }$ and $\mathrm{AUC}_{\text {inf }}$ were increased in the presence of ketoconazole administered at a dosage of $400 \mathrm{mg}$ once daily compared with amenamevir 


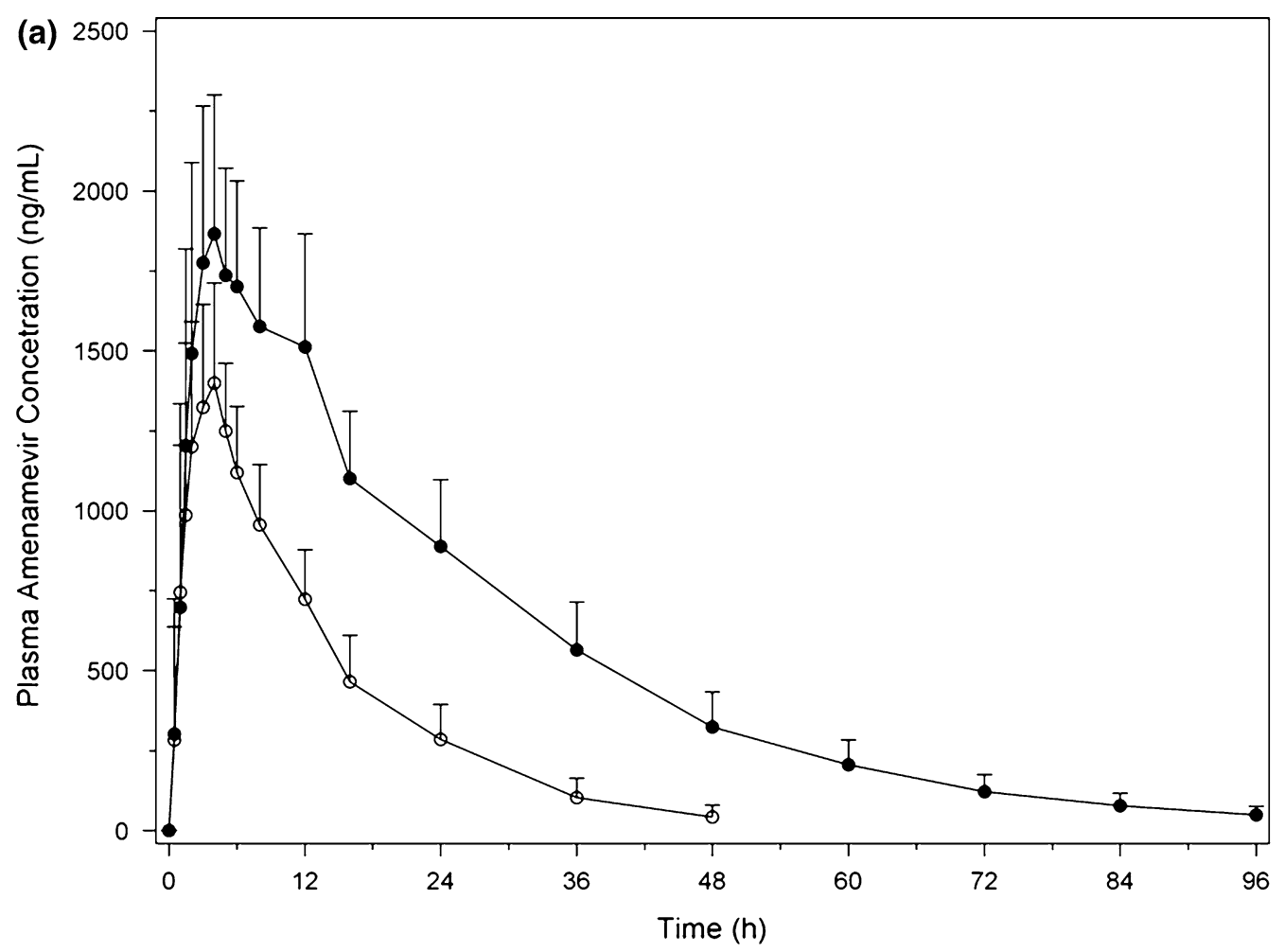

(b)

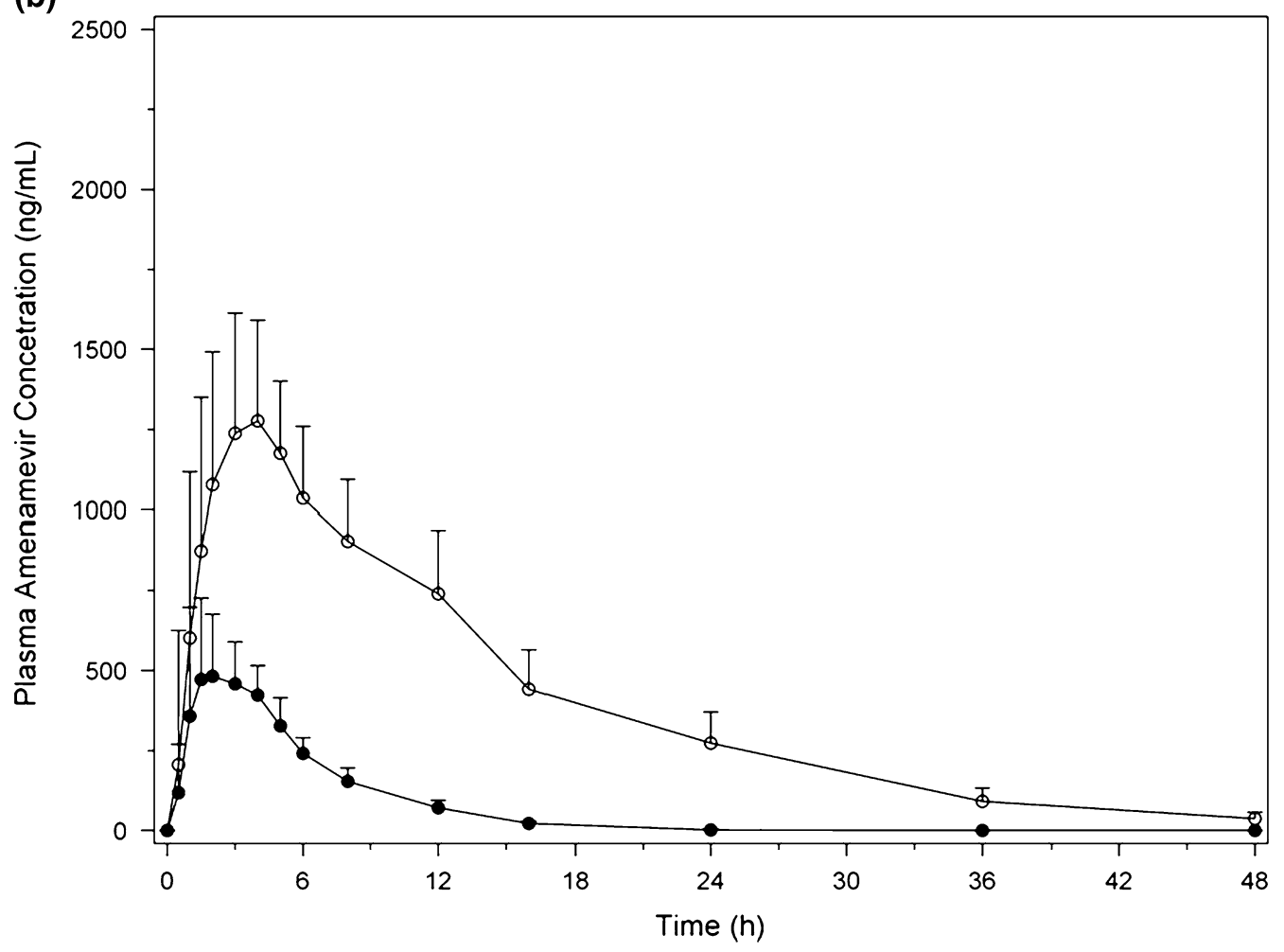


alone. The plasma concentration versus time profile for amenamevir alone and with ketoconazole is shown in Fig. 1a. The amenamevir $t_{\max }$ showed no difference between the absence or presence of ketoconazole. However, with ketoconazole coadministration, the mean $t_{1 / 2}$ for amenamevir increased 2.2-fold, while CL/F decreased 2.6-fold. A summary of amenamevir pharmacokinetics in the absence and presence of ketoconazole is presented in Table 2 .

On the basis of the LS GMR of amenamevir plus ketoconazole versus amenamevir alone for $C_{\max }$ of 1.30 (90\% CI 1.17-1.45), the null hypothesis of a relevant interaction between amenamevir and ketoconazole was not rejected as the $90 \% \mathrm{CI}$ for the $C_{\max }$ LS GMR was not within the predefined limits. For the LS GMR for $\mathrm{AUC}_{\mathrm{inf}}$ of 2.58 (90\% CI 2.32-2.87), the existence of a significant interaction between amenamevir and ketoconazole was confirmed as the entire $90 \% \mathrm{CI}$ was above the predefined upper limit.

\section{Effects of Rifampicin on Amenamevir Pharmacokinetics}

After a single 400-mg dose of amenamevir in study 15L-CL-009, the amenamevir mean $C_{\max }$ and $\mathrm{AUC}_{\text {inf }}$ were decreased during administration of rifampicin at a dosage of $600 \mathrm{mg}$ once daily compared with amenamevir alone. The amenamevir mean $t_{\max }$ also tended to decrease during rifampicin coadministration. The plasma concentration versus time profile for amenamevir alone and with rifampicin is shown in Fig. 1b. In the presence of rifampicin, the amenamevir mean $t_{1 / 2}$ decreased 2.7-fold and the amenamevir mean $\mathrm{CL} / \mathrm{F}$ increased sixfold compared with amenamevir alone. A summary

Table 2 Summary of amenamevir pharmacokinetic parameters after administration of a single 400-mg dose of amenamevir alone and with ketoconazole at a dosage of $400 \mathrm{mg}$ once daily or rifampicin at a dosage of $600 \mathrm{mg}$ once daily

\begin{tabular}{|c|c|c|c|c|}
\hline \multirow[t]{3}{*}{ Parameter } & \multicolumn{4}{|l|}{ Study } \\
\hline & \multicolumn{2}{|l|}{ 15L-CL-008 } & \multicolumn{2}{|l|}{ 15L-CL-009 } \\
\hline & $\begin{array}{l}\text { Amenamevir alone } \\
(n=22)\end{array}$ & $\begin{array}{l}\text { Amenamevir + ketoconazole } \\
(n=22)\end{array}$ & $\begin{array}{l}\text { Amenamevir alone } \\
(n=22)\end{array}$ & $\begin{array}{l}\text { Amenamevir }+ \text { rifampicin } \\
(n=22)\end{array}$ \\
\hline $\begin{array}{l}\mathrm{AUC}_{\text {inf }} \\
\quad(\mathrm{ng} \mathrm{h} / \mathrm{mL})^{\mathrm{a}}\end{array}$ & $19,917 \pm 5561$ & $51,483 \pm 12048$ & $18,658 \pm 4935$ & $3115 \pm 930$ \\
\hline $\begin{array}{l}C_{\max } \\
\quad(\mathrm{ng} / \mathrm{mL})^{\mathrm{a}}\end{array}$ & $1448 \pm 329$ & $1886 \pm 448$ & $1325 \pm 282$ & $563 \pm 218$ \\
\hline$t_{\max }(\mathrm{h})^{\mathrm{b}}$ & $4.0(1.0-5.0)$ & $4.0(3.0-6.0)$ & $3.0(0.5-5.0)$ & $1.75(1.0-5.0)$ \\
\hline$t_{1 / 2}(\mathrm{~h})$ & $8.1 \pm 1.9$ & $17.5 \pm 2.9$ & $8.0 \pm 1.2$ & $2.9 \pm 0.6$ \\
\hline $\mathrm{CL} / \mathrm{F}(\mathrm{L} / \mathrm{h})$ & $20.8 \pm 5.9$ & $8.0 \pm 2.1$ & $22.1 \pm 5.9$ & $133.5 \pm 40.0$ \\
\hline LS GMR ${ }^{c}$ & \multicolumn{2}{|c|}{ Amenamevir + ketoconazole vs amenamevir alone } & \multicolumn{2}{|c|}{ Amenamevir + rifampicin vs amenamevir alone } \\
\hline $\mathrm{AUC}_{\text {inf }}$ & \multicolumn{2}{|l|}{$2.58(2.32-2.87)$} & \multicolumn{2}{|l|}{$0.17(0.15-0.19)$} \\
\hline$C_{\max }$ & \multicolumn{2}{|l|}{$1.30(1.17-1.45)$} & \multicolumn{2}{|l|}{$0.42(0.37-0.49)$} \\
\hline \multicolumn{5}{|c|}{$\begin{array}{l}\text { All values are presented as the arithmetic mean } \pm \text { standard deviation } \\
A U C_{i n f} \text { area under the plasma concentration versus time curve from ti } \\
\text { plasma concentration, } L S G M R \text { least squares geometric mean ratio, } t_{1 / 2} \\
\text { a Values are presented as the geometric mean } \pm \text { standard deviation } \\
\text { b Values are presented as the median, with the range in parentheses } \\
\text { c The } 90 \% \text { confidence interval is given in parentheses }\end{array}$} \\
\hline
\end{tabular}




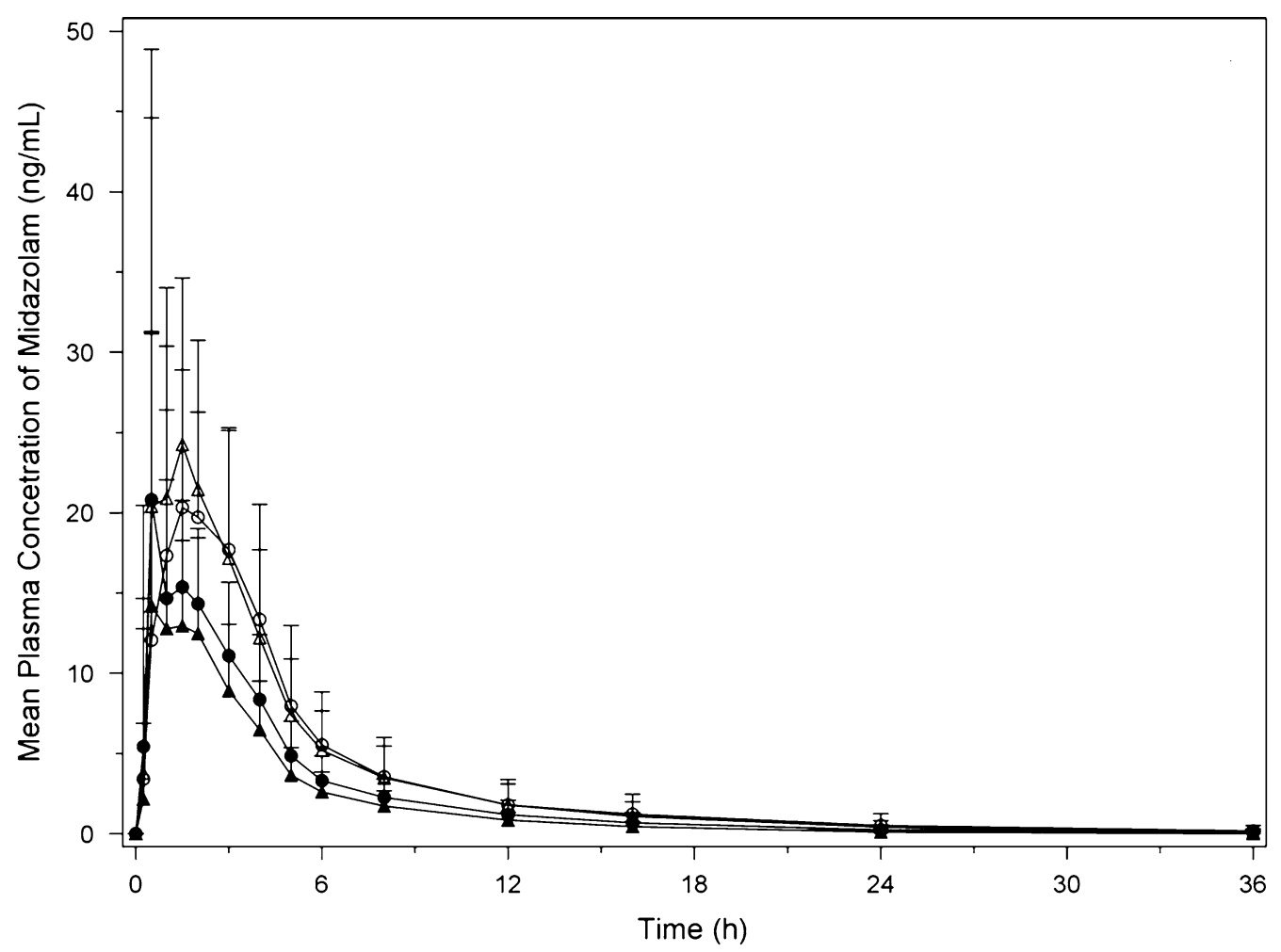

Fig. 2 Mean midazolam plasma concentration versus time curve after administration of a single $7.5-\mathrm{mg}$ dose of midazolam either alone (open circles) and with amenamevir at a dosage of $200 \mathrm{mg}$ once daily (closed circles) or

of the amenamevir pharmacokinetics in the absence and presence of rifampicin is presented in Table 2.

On the basis of the LS GMR of amenamevir plus rifampicin versus amenamevir alone for both $C_{\max }$ and $\mathrm{AUC}_{\mathrm{inf}}$ of $0.42 \quad(90 \% \mathrm{CI}$ 0.37-0.49) and 0.17 (90\% CI 0.15-0.19), respectively, a significant interaction between amenamevir and rifampicin was confirmed as the $90 \%$ CIs for both parameters were entirely outside the predefined limits.

\section{Effects of Amenamevir on Midazolam Pharmacokinetics}

In study 15L-CL-010, multiple doses of amenamevir (200 and $400 \mathrm{mg}$ ) once daily both affected the pharmacokinetics of a single $7.5-\mathrm{mg}$ dose of midazolam, with the 400-mg dose showing the most pronounced effects. After a single dose of midazolam, the midazolam mean $\mathrm{AUC}_{\mathrm{inf}}$ and $C_{\max }$ decreased during alone (open triangles) and with amenamevir at a dosage of $400 \mathrm{mg}$ once daily (closed triangles)

administration of amenamevir at a dosage of $400 \mathrm{mg}$ once daily compared with midazolam alone, although during administration of amenamevir at a dosage of $200 \mathrm{mg}$ once daily, only the midazolam $\mathrm{AUC}_{\mathrm{inf}}$ decreased. The plasma concentration versus time profile for midazolam after administration of midazolam alone and with amenamevir at dosages of 200 and $400 \mathrm{mg}$ once daily is shown in Fig. 2. The changes in midazolam exposure were associated with a reduction in midazolam mean $t_{1 / 2}$ and an increase in mean CL/F. A summary of the midazolam pharmacokinetics in the absence and presence of amenamevir at a dosage of 200 or $400 \mathrm{mg}$ once daily is presented in Table 3.

On the basis of the LS GMR for $\mathrm{AUC}_{\text {inf }}(0.72)$, the amenamevir 200-mg dose shows a significant interaction with midazolam, as the $90 \%$ CI for this ratio (0.65-0.79) was entirely outside the predefined limits. On the basis of the LS GMRs for both $C_{\max }$ and $\mathrm{AUC}_{\text {inf }}$ of $0.63(90 \% \mathrm{CI}$ 
Table 3 Summary of midazolam pharmacokinetic parameters after administration of a single 7.5-mg dose of midazolam alone and with amenamevir at a dosage of 200 or $400 \mathrm{mg}$ once daily in study 15L-CL-010

\begin{tabular}{|c|c|c|c|c|}
\hline \multirow[t]{2}{*}{ Parameter } & \multicolumn{2}{|c|}{ Amenamevir 200 mg } & \multicolumn{2}{|c|}{ Amenamevir $400 \mathrm{mg}$} \\
\hline & $\begin{array}{l}\text { Midazolam alone } \\
(n=22)\end{array}$ & $\begin{array}{l}\text { Midazolam + amenamevir } \\
(n=22)\end{array}$ & $\begin{array}{l}\text { Midazolam alone } \\
(n=22)\end{array}$ & $\begin{array}{l}\text { Midazolam + amenamevir } \\
(n=22)\end{array}$ \\
\hline $\begin{array}{l}\mathrm{AUC}_{\text {inf }}(\mathrm{ng} \mathrm{h} / \\
\mathrm{mL})^{\mathrm{a}}\end{array}$ & $104.5 \pm 64.7$ & $74.8 \pm 37.5$ & $107.5 \pm 55.8$ & $57.3 \pm 25.7$ \\
\hline $\begin{array}{c}C_{\max }(\mathrm{ng} / \\
\mathrm{mL})^{\mathrm{a}}\end{array}$ & $26.3 \pm 13.7$ & $25.9 \pm 19.1$ & $30.2 \pm 22.7$ & $19.0 \pm 12.9$ \\
\hline$t_{\max }(\mathrm{h})^{\mathrm{b}}$ & $1.5(0.5-3.0)$ & $1.0(0.3-4.0)$ & $1.5(0.3-3.0)$ & $1.0(0.5-4.0)$ \\
\hline$t_{1 / 2}(\mathrm{~h})$ & $5.7 \pm 2.5$ & $4.8 \pm 1.9$ & $5.3 \pm 1.8$ & $4.3 \pm 1.4$ \\
\hline $\mathrm{CL} / \mathrm{F}(\mathrm{L} / \mathrm{h})$ & $80.1 \pm 37.0$ & $109.0 \pm 43.4$ & $78.2 \pm 39.2$ & $143.8 \pm 68.0$ \\
\hline LS GMR ${ }^{c}$ & \multicolumn{2}{|c|}{$\begin{array}{l}\text { Midazolam + amenamevir } 200 \mathrm{mg} \text { vs midazolam } \\
\text { alone }\end{array}$} & \multicolumn{2}{|c|}{$\begin{array}{l}\text { Midazolam + amenamevir } 400 \mathrm{mg} \text { vs midazolam } \\
\text { alone }\end{array}$} \\
\hline $\mathrm{AUC}_{\mathrm{inf}}$ & \multicolumn{2}{|l|}{$0.72(0.65-0.79)$} & \multicolumn{2}{|l|}{$0.53(0.47-0.61)$} \\
\hline$C_{\max }$ & \multicolumn{2}{|l|}{$0.98(0.82-1.18)$} & \multicolumn{2}{|l|}{$0.63(0.50-0.80)$} \\
\hline
\end{tabular}

All values are presented as the arithmetic mean \pm standard deviation unless stated otherwise

$A U C_{\text {inf }}$ area under the plasma concentration versus time curve from time zero to infinity, $C L / F$ clearance, $C_{\text {max }}$ maximum plasma concentration, $L S G M R$ least squares geometric mean ratio, $t_{1 / 2}$ half-life, $t_{\max }$ time to maximum plasma concentration

${ }^{a}$ Values are presented as the geometric mean \pm standard deviation

${ }^{\mathrm{b}}$ Values are presented as the median, with the range in parentheses

c The $90 \%$ confidence interval is given in parentheses

$0.50-0.80)$ and $0.53 \quad(90 \%$ CI $0.47-0.61)$, respectively, which both have $90 \%$ CIs that were entirely outside the predefined limits, a significant interaction occurred between amenamevir at a dosage of $400 \mathrm{mg}$ once daily and midazolam. The LS GMR of an amenamevir dose of $400 \mathrm{mg}$ versus an amenamevir dose of $200 \mathrm{mg}$ was $0.64(90 \% \mathrm{CI} 0.48-0.86)$ for $C_{\max }$ and 0.75 (90\% CI $0.63-0.88)$ for $\mathrm{AUC}_{\text {inf }}$.

\section{Effects of Amenamevir on Warfarin Pharmacokinetics}

After a single 25-mg dose of warfarin in study 15L-CL-018, the $(S)$-warfarin and $(R)$-warfarin mean $C_{\max }$ values were slightly higher and the $\mathrm{AUC}_{\text {inf }}$ values were slightly lower during administration of amenamevir at a dosage of 200 or $400 \mathrm{mg}$ once daily compared with warfarin alone. However, as the $90 \%$ CIs of the LS GMRs for these parameters remained within the predefined limits, there was no significant pharmacokinetic interaction between amenamevir and $(S)$-warfarin or $(R)$-warfarin.

The mean (S)-warfarin $t_{1 / 2}$ and $\mathrm{CL} / \mathrm{F}$ values were similar during administration of both doses of amenamevir compared with warfarin alone. The mean (S)-warfarin $t_{\max }$ was similar to that of warfarin plus amenamevir at dose of $200 \mathrm{mg}$ and warfarin alone, but tended to be lower with warfarin plus amenamevir at a dose of $400 \mathrm{mg}$ versus warfarin alone. The mean $(R)$-warfarin $t_{1 / 2}$ values in the presence of amenamevir at a dosage of 200 or $400 \mathrm{mg}$ once daily were slightly lower than those observed with warfarin alone, while the mean $(R)$-warfarin $\mathrm{CL} / \mathrm{F}$ and $t_{\max }$ values remained similar both in the presence and in absence of amenamevir. A summary of the (S)-warfarin and $(R)$-warfarin pharmacokinetic parameters in the absence and presence of amenamevir at a dosage of 200 or $400 \mathrm{mg}$ once daily is presented in Table 4. 


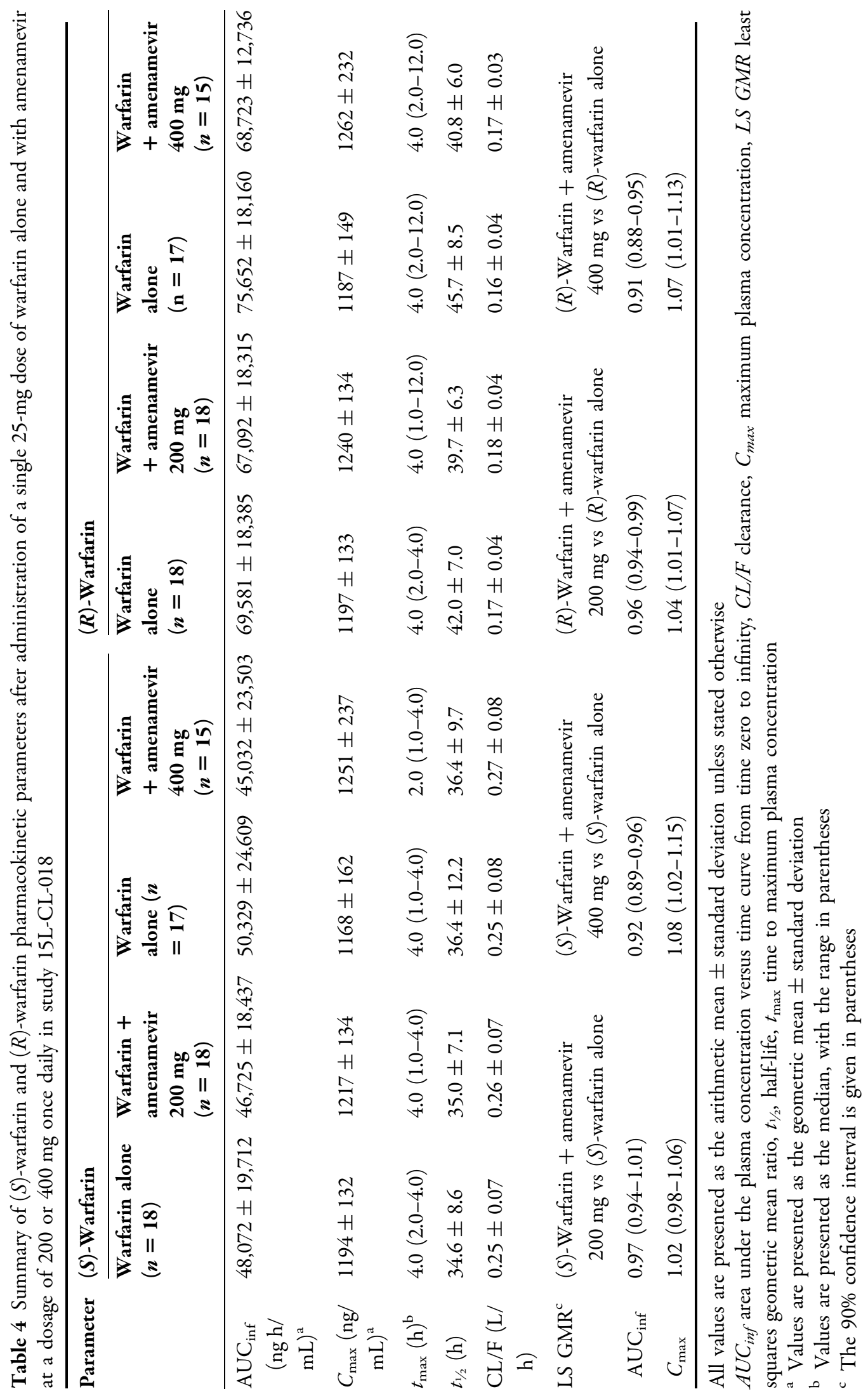


Table 5 Summary of warfarin pharmacodynamic (prothrombin time, PT) parameters after administration of a single 25-mg dose of warfarin alone and with amenamevir at a dosage of 200 or $400 \mathrm{mg}$ once daily in study 15L-CL-018

\begin{tabular}{|c|c|c|c|c|}
\hline \multirow[t]{2}{*}{ Parameter } & \multicolumn{2}{|c|}{ Amenamevir $200 \mathrm{mg}$} & \multicolumn{2}{|c|}{ Amenamevir $400 \mathrm{mg}$} \\
\hline & $\begin{array}{l}\text { Warfarin alone } \\
(n=18)\end{array}$ & $\begin{array}{l}\text { Warfarin + amenamevir } \\
200 \mathrm{mg}(n=18)\end{array}$ & $\begin{array}{l}\text { Warfarin alone } \\
(n=17)\end{array}$ & $\begin{array}{l}\text { Warfarin + amenamevir } \\
400 \mathrm{mg}(n=15)\end{array}$ \\
\hline $\begin{array}{l}\mathrm{AUC}_{\mathrm{PT} ; 0-168 \mathrm{~h}} \\
\quad(\mathrm{~s} \mathrm{~h})^{\mathrm{a}}\end{array}$ & $2480 \pm 246$ & $2411 \pm 199$ & $2556 \pm 254$ & $2425 \pm 163$ \\
\hline $\mathrm{PT}_{\max }(\mathrm{s})$ & $17.2 \pm 2.3$ & $17.3 \pm 2.5$ & $17.9 \pm 2.4$ & $16.7 \pm 1.9$ \\
\hline $\mathrm{tPT}_{\max }(\mathrm{h})$ & $36.0 \pm 12.4$ & $35.9 \pm 12.3$ & $39.5 \pm 14.6$ & $36.0 \pm 13.6$ \\
\hline LS mean ratio ${ }^{b}$ & \multicolumn{2}{|c|}{ Warfarin + amenamevir $200 \mathrm{mg}$ vs warfarin alone } & \multicolumn{2}{|c|}{ Warfarin + amenamevir $400 \mathrm{mg}$ vs warfarin alone } \\
\hline $\mathrm{AUC}_{\mathrm{PT} ; 0-168 \mathrm{~h}}$ & \multicolumn{2}{|l|}{$0.97(0.94-1.00)$} & \multicolumn{2}{|l|}{$0.96(0.95-0.98)$} \\
\hline $\mathrm{PT}_{\max }$ & \multicolumn{2}{|l|}{$1.00(0.96-1.04)$} & \multicolumn{2}{|l|}{$0.95(0.93-0.97)$} \\
\hline
\end{tabular}

All values are presented as the arithmetic mean \pm standard deviation unless stated otherwise

$A U C_{P T ; 0-168 \mathrm{~h}}$ area under the prothrombin time versus time curve from time zero to $168 \mathrm{~h}, L S$ least squares, $P T_{\text {max }}$ maximum prothrombin time, $t P T_{\max }$ time to maximum prothrombin time

a Values are presented as the geometric mean \pm standard deviation

b The $90 \%$ confidence interval is given in parentheses

\section{Pharmacodynamics}

\section{Effects of Amenamevir on Warfarin Pharmacodynamics}

After a single dose of warfarin, mean $\mathrm{PT}_{\max }$ values were slightly lower during administration of amenamevir at a dosage of $400 \mathrm{mg}$ once daily and were reached slightly earlier with both amenamevir doses than with warfarin alone. Compared with warfarin alone,

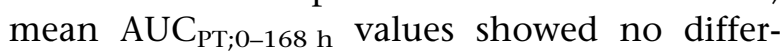
ence with amenamevir at a dosage of $200 \mathrm{mg}$ once daily and were slightly lower with amenamevir at a dosage of $400 \mathrm{mg}$ once daily. On the basis of the LS mean ratios for $\mathrm{PT}_{\max }$ and

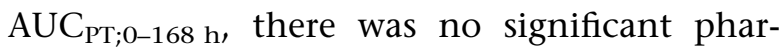
macodynamic interaction between amenamevir and warfarin, as the $90 \%$ CIs for both ratios remained within the predefined limits for both doses of amenamevir. A summary of the warfarin pharmacodynamic parameters in the absence and presence of amenamevir at a dosage of 200 or $400 \mathrm{mg}$ once daily is presented in Table 5.

\section{Safety}

Across all four studies, there were no deaths, serious TEAEs, or TEAEs that resulted in discontinuation of participation in the study. In addition, no clinically significant changes from the baseline were observed in laboratory parameters, vital signs, or 12-lead ECG recordings, and physical examination findings remained normal.

In study 15L-CL-008, 13 TEAEs were reported by eight participants (36.4\%). Headache was the only TEAE reported by more than one participant (five participants in total; 22.7\%), and was reported during administration of ketoconazole alone (four participants; 18.2\%) and amenamevir plus ketoconazole (one participant; $4.5 \%$ ). All cases of headache were considered to be TEAEs related to the study drug.

In study 15L-CL-009, four participants (18.2\%) reported four TEAEs overall. The only TEAE to be reported by more than one participant (two participants in total; 9,1\%) was flatulence. All TEAEs in this study were considered by the investigator to be related to the study drug. 
In study 15L-CL-010, TEAEs were reported by two participants $(9.1 \%)$ in the group that received the 200-mg dose of amenamevir and by two participants $(9.1 \%)$ in group that received the 400-mg dose of amenamevir. None of the TEAEs were reported by more than one participant. Palpitations and asthenia (midazolam plus amenamevir at a dose of $400 \mathrm{mg}$ ) were TEAEs that were considered to be related to the study drug.

In study 15L-CL-018, six TEAEs were reported by five participants $(27.8 \%)$ in the group that received amenamevir at a dose of $200 \mathrm{mg}$, and six TEAEs were reported by three participants $(15.8 \%)$ in the group that received amenamevir at a dose of $400 \mathrm{mg}$. None of the TEAEs were reported by more than one participant. In this study, eight TEAEs were considered to be related to the study drug: two TEAEs with warfarin alone (nausea and dyspepsia), four TEAEs with amenamevir alone (myalgia with the 200-mg dose, and back pain, abdominal pain, and dyspepsia with the 400-mg dose), and two TEAEs with warfarin plus amenamevir (headache with 200-mg amenamevir dose and oral herpes with the 400-mg amenamevir dose).

\section{DISCUSSION}

The viral helicase inhibitor amenamevir is a nonnucleoside anti-HHV drug available for the treatment of VZV infections. On the basis of in vitro data, amenamevir is thought to be predominantly metabolized by CYP3A $4 / 5$, and is a potential inducer of CYP3A4 and CYP2C9 (unpublished data). However, no clinical drug-drug interaction studies have previously been conducted.

Data from the four clinical studies in healthy humans described here confirm the existence of interactions between amenamevir and ketoconazole (a strong CYP3A4 inhibitor) and between amenamevir and rifampicin (a strong CYP3A4 inducer). Amenamevir exposure was significantly increased during coadministration with ketoconazole and significantly decreased during coadministration with rifampicin. Given that amenamevir is known to be a CYP3A4 substrate, the effects of ketoconazole and rifampicin on the pharmacokinetic profile of amenamevir were to be expected.

In these clinical studies, a significant interaction between amenamevir and midazolam (a CYP3A4 substrate) was also confirmed, with significant decreases in midazolam exposure when a single 7.5-mg dose of midazolam was coadministered with amenamevir at a dosage of $400 \mathrm{mg}$ once daily compared with midazolam alone. This pharmacokinetic interaction with amenamevir appears to be related to the dose, with numerically greater decreases in midazolam exposure with $400 \mathrm{mg}$ versus $200 \mathrm{mg}$ once daily administration of amenamevir. The interaction between amenamevir and midazolam confirms that amenamevir is a CYP3A4 enzyme inducer.

In these studies, no significant pharmacokinetic or pharmacodynamic interactions were observed between amenamevir and $(S)$-warfarin (a CYP2C9 substrate). In contrast to in vitro data, which suggested that amenamevir is a potential CYP2C9 inducer (unpublished data), data from these human studies indicate that amenamevir is not an inducer of CYP2C9.

Amenamevir was safe and generally well tolerated in these studies, with no deaths or serious adverse events reported and no clinically significant changes in vital signs, clinical laboratory parameters, or ECG recordings. These observations are consistent with those of previous clinical studies of amenamevir in patients with recurrent genital herpes infection [13].

These studies have some limitations that may affect the interpretation of the findings. As with most drug-drug interaction studies, the study populations were relatively small, and randomized controlled studies with larger patient populations are needed to confirm the safety and tolerability of amenamevir in clinical practice.

\section{CONCLUSIONS}

These studies confirm that amenamevir (as a CYP3A4 substrate) can interact with ketoconazole or rifampicin, and (as a CYP3A4 inducer) can interact with midazolam in a 
dose-dependent fashion; however, no significant interaction was observed between amenamevir and (S)-warfarin, indicating that amenamevir may not be an inducer of CYP2C9. Information regarding potential interactions between amenamevir and other drugs is particularly important in elderly or immunocompromised patient populations (who may be receiving treatment with other drugs), and appropriate caution will be needed in patients receiving concomitant medications. The recommended dose of amenamevir for VZV infection is $400 \mathrm{mg} /$ day by oral administration for 7 days. Because of this, the drug interactions seen in these studies may not be a significant issue to manage in the clinical setting, as the amenamevir administration period is relatively short. However, appropriate caution will be needed in the following situations:

1. CYP3A4 substrates: CYP3A4 substrates should be used with caution because of a potential decrease in their exposure.

2. CYP3A4 inducers: Amenamevir and strong CYP3A4 inducers should not be coadministered. Amenamevir exposure was demonstrated to decrease, which could possibly result in reduction or loss of antiviral efficacy.

3. CYP3A4 inhibitors: Strong CYP3A4 inhibitors should be used with caution because of a potential increase in amenamevir exposure.

\section{ACKNOWLEDGEMENTS}

Sponsorship for the studies, article processing charges, and open access fee were funded by Astellas Pharma. All authors had full access to all of the data in these studies and take complete responsibility for the integrity of the data and accuracy of the data analysis. We thank Sarah Grieg of Springer Healthcare Communications who wrote the first draft of the manuscript. This medical writing assistance was funded by Astellas Pharma. All named authors meet the International Committee of Medical Journal Editors (ICMJE) criteria for authorship for the manuscript, take responsibility for the integrity of the work as a whole, and have given final approval for the version to be published.

Disclosures. Tomohiro Kusawake is an employee of Astellas Pharma. Martin den Adel is an employee of Astellas Pharma. Dorien Groenendaal-van de Meent is an employee of Astellas Pharma. Alberto Garcia-Hernandez is an employee of Astellas Pharma. Akitsugu Takada is an employee of Astellas Pharma. Kota Kato is an employee of Astellas Pharma. Yoshiaki Ohtsu is an employee of Astellas Pharma. Masataka Katashima is an employee of Astellas Pharma. The authors have no other relevant affiliations or financial involvement with any organization or entity with a financial interest in, or a financial conflict with, the subject matter or materials discussed in the article other than those disclosed.

Compliance with Ethics Guidelines. The study protocols were approved by the institutional review boards at the study site [Hôpital Ambroise-Paré, Secrétariat du CPP Ile de France VIII, Laboratoire d'Anatomopathologie (studies 15L-CL-008 and 15L-CL-009) and CPP Ile de France I, Secrétariat du CPP Ile de France I, Hôpital Hotel-Dieu (studies 15L-CL-010 and 15L-CL-018)]. All procedures followed were in accordance with the ethical standards of the responsible committee on human experimentation (institutional and national) and with the Helsinki Declaration of 1964, as revised in 2000. Informed consent was obtained from all participants for their being included in the study.

Data Availability. The datasets generated during and/or analyzed during the current study are not publicly available because these studies were small single-center studies and anonymization of these data is difficult to achieve.

Open Access. This article is distributed under the terms of the Creative Commons Attribution-NonCommercial 4.0 International License (http://creativecommons.org/licenses/ by-nc/4.0/), which permits any noncommercial use, distribution, and reproduction in any medium, provided you give appropriate credit 
to the original author(s) and the source, provide a link to the Creative Commons license, and indicate if changes were made.

\section{REFERENCES}

1. Arvin AM. Varicella-zoster virus. Clin Microbiol Rev. 1996;9(3):361-81.

2. Gilden D, Mahalingam R, Nagel MA, Pugazhenthi S, Cohrs RJ. Review: the neurobiology of varicella zoster virus infection. Neuropathol Appl Neurobiol. 2011;37(5):441-63.

3. Kawai K, Gebremeskel BG, Acosta CJ. Systematic review of incidence and complications of herpes zoster: towards a global perspective. BMJ Open. 2014;4(6):e004833.

4. GlaxoSmithKline. ZOVIRAX ${ }^{\circledR}$ (acyclovir) capsules, tablets, suspension: prescribing information. 2005. https://www.accessdata.fda.gov/drugsatfda_docs/ label/2005/018828s030,020089s019,019909s020lbl. pdf. Accessed 21 Jul 2017.

5. GlaxoSmithKline. $\quad$ VALTREX $^{\circledR} \quad$ (valacyclovir hydrochloride) caplets: prescribing information. 2008. https://www.accessdata.fda.gov/drugsatfda docs/label/2008/020487s014lbl.pdf. Accessed 21 Jul 2017.

6. Novartis. Famvir ${ }^{\circledR}$ (famciclovir) tablets: prescribing information. 2006. https://www.accessdata.fda. gov/drugsatfda_docs/label/2006/020363s026lbl.pdf. Accessed 21 July 2017.
7. Chono K, Katsumata K, Kontani T, et al. ASP2151, a novel helicase-primase inhibitor, possesses antiviral activity against varicella-zoster virus and herpes simplex virus types 1 and 2 . J Antimicrob Chemother. 2010;65(8):1733-41.

8. Himaki T, Masui Y, Chono K, et al. Efficacy of ASP2151, a helicase-primase inhibitor, against thymidine kinase-deficient herpes simplex virus type 2 infection in vitro and in vivo. Antiviral Res. 2012;93(2):301-4.

9. Ohtsu Y, van Trigt R, Takama K, et al. Quantification of ASP2151 in human plasma and urine: a pitfall associated with supersaturation of analyte in urine. Chromatographia. 2017;80(2):217-27.

10. Katsumata K, Chono K, Kato K, et al. Pharmacokinetics and pharmacodynamics of ASP2151, a helicase-primase inhibitor, in a murine model of herpes simplex virus infection. Antimicrob Agents Chemother. 2013;57(3):1339-46.

11. Johnson RW, Alvarez-Pasquin MJ, Bijl M, et al. Herpes zoster epidemiology, management, and disease and economic burden in Europe: a multidisciplinary perspective. Ther Adv Vaccines. 2015;3(4):109-20.

12. Kusawake T, Keirns J, Kowalski D, et al. Pharmacokinetics and safety of amenamevir in healthy subjects: analysis of four randomized phase 1 studies. Advances in Therapy (under review).

13. Tyring S, Wald A, Zadeikis N, Dhadda S, Takenouchi K, Rorig R. ASP2151 for the treatment of genital herpes: a randomized, double-blind, placebo- and valacyclovir-controlled, dose-finding study. J Infect Dis. 2012;205(7):1100-10. 